\title{
Valor del PET/CT semicuantitativo (SUVmax) en el estudio de nódulo pulmonar sólido
}

\author{
DAVID LADRÓN DE GUEVARA H.*, PAOLO CATALÁN V.*, CLAUDIA HERNÁNDEZ M.*, y \\ JUAN PABLO ZHINDON P.**
}

\section{Value of Semiquantitative PET/CT (SUVmax) in study of solid lung nodule}

Aim: To establish the diagnostic accuracy of PET/CT in study of solid lung nodule (LN) using SUVmax index. Method: We revised PET/CT data base, selecting those scans asked to evaluate a solid $L N$. Only confirmed malign o benign LN were included. Subsolid LN, lung masses (> $3 \mathrm{~cm}$ ), and known or suspected lung metastases were excluded. SUVmax was measured in each LN, and best cutoff for malignant and benign lesion was calculated. Results: Of the whole group of $140 \mathrm{LN}, 60 \%$ were confirmed as malignant, and $40 \%$ as benign (100\% and 59,6\% of histological confirmation, respectively). SUVmax $\leq 1,0$ showed sensibility of $98,8 \%$, negative predictive value (NPV) of 96,2\%, and negative likelihood ratio (LR -) of 0,027. SUVmax $\leq 2,5$ was not able to guarantee reasonably benign nature of $L N$, showing NPV of 69,4\% and LR - of 0,295. SUVmax > 2,5 and > 5,0 was associated to malign lesion in $83 \%$ and $93 \%$ of cases, respectively (LR + of 3,333 and 8,889). Conclusion: PET/CT shows high accuracy estimating the nature of solid LN. SUVmax $\leq 1,0$ is highly predictive of benignity, and SUVmax $\geq 2,5$ is highly predictive of malignancy. SUVmax values between 1,0 and 2,5 were not able to characterize efficiently $L N$.

Key words: Lung nodule; pulmonary nodule; lung neoplasm; positron emission tomography; computed tomography; radionuclide imaging.

\section{Resumen}

Objetivo: Determinar el rendimiento diagnóstico del PET/CT en el estudio de nódulo pulmonar (NP) utilizando SUVmax. Método: Se revisó la base de datos de PET/CT, seleccionando aquellos solicitados para estudio de NP sólido. Se incluyeron sólo aquellos NP confirmados como malignos o benignos. Se excluyó NP subsólidos, masas pulmonares (>3 cm), y pacientes con metástasis conocidas. Se midió SUVmax de las lesiones, determinando mejores valores de corte para malignidad y benignidad. Resultados: De los 140 NP estudiados, el 60\% (84/140) fueron confirmados como malignos y el $40 \%$ como benignos (100\% y 59,6\% de confirmación histológica, respectivamente). Un SUVmax $\leq 1,0$ mostró sensibilidad 98,8\%, valor predictivo negativo (VPN) 96,2\%, y Likelihood ratio negativo (LR -) 0,027. Un SUVmax $\leq 2,5$ no fue capaz de asegurar razonablemente benignidad con VPN 69,4\%, y LR - 0,295. Valores de $S U V>2,5$ y 5,0 se asociaron a malignidad en $83 \%$ y 93\% de los casos, respectivamente (LR+ 3,333 y 8,889). Conclusión: El PET/CT presenta alto rendimiento diagnóstico en estimar la naturaleza de un NP. Un valor de SUVmax $\leq 1$ es altamente predictivo de benignidad, y un valor de $S U V \max \geq 2,5$ de malignidad. Valores entre 1,0 y 2,5 no permiten caracterizar eficientemente los NP.

Palabras clave: Nódulo pulmonar; neoplasia pulmonar; PET-CT; tomografía computarizada; imagenología radionuclear.

* Departamento de Radiología, Clínica Las Condes, Santiago, Chile.

** Departamento de Medicina Nuclear, Instituto Nacional del Cáncer, Santiago, Chile. 


\section{Introducción}

El nódulo pulmonar (NP) es una entidad de relativa frecuencia en la práctica clínica, tanto en el contexto de programas de pesquisa de cáncer pulmonar donde se realiza radiografía de tórax o Tomografía Computada (TC) a población de riesgo, como en pacientes estudiados por otra causa donde el NP constituye un hallazgo incidental. Se estima que entre 25 y $50 \%$ de los adultos sanos tendrá uno o varios NP visibles en la $\mathrm{TC}^{1}$.

Decidir como enfrentar este hallazgo desde el punto de vista clínico no es tarea fácil, y posiblemente lo primero que debiera hacerse es realizar una aproximación al riesgo de malignidad de la lesión, con el objetivo de decidir entre cirugía, biopsia no quirúrgica, o seguimiento con $\mathrm{TC}^{2}$.

Para estimar la probabilidad de malignidad de un NP se deben considerar diversos factores clínicos como la edad, antecedente tabáquico, antecedentes familiares de cáncer, y ciertas características del NP a la TC como su ubicación en el pulmón, tamaño, morfología (márgenes, densidad), y realce tras la administración de contraste iodado ${ }^{1,2,3}$.

Según el American College of Chest Physicians, los NP con muy baja probabilidad de malignidad $(<5 \%$ riesgo estimado de cáncer) debieran ser controlados con TC, y aquellos con alta probabilidad ( $>65 \%$ riesgo estimado) sometidos a cirugía ${ }^{2}$. Los nódulos con probabilidad baja/media (5-65\% riesgo estimado) requerirán otro método diagnóstico para decidir el manejo.

Uno de estos métodos es el PET/CT, un examen híbrido, funcional y morfológico, que permite estimar la captación de un determinado radiotrazador en una lesión. El radiotrazador más utilizado es la glucosa unida a Flúor18, un isótopo emisor de positrones, formando la molécula F18-Fluorodeoxiglucosa (F18-FDG). La intensidad de captación de F18-FDG en una determinada lesión se relaciona a su grado de actividad metabólica y su potencial de maligni$\mathrm{dad}^{4}$. Mientras mayor captación muestre, mayor probabilidad de que sea maligna.

El PET/CT con F18-FDG se ha usado ampliamente en el estudio de nódulos pulmonares, con diversos resultados que van desde adecuado costo beneficio del método ${ }^{5}$ hasta poca utilidad clínica con bajo rendimiento diagnóstico ${ }^{6,7}$. Un metaanálisis publicado el año 2018 describe una sensibilidad de 0,89 (IC 95\%, 0,87-0,91) y una especificidad de 0,70 (IC 95\%, 0,66-0,73), y concluye que su rendimiento diagnóstico es insuficiente para estudiar nódulos pulmonares ${ }^{8}$. Otro metaanálisis igualmente reciente describe sensi- bilidad de $0,82(95 \% \mathrm{CI}, 0,76-0,87)$ y especificidad de 0,81 (95\% CI, 0,66 - 0,90), y concluye que el PET/CT es una útil herramienta en la detección de NP malignos ${ }^{9}$. Ambos metaanálisis incluyeron casi exclusivamente estudios que cuantificaron la captación de los NP. De hecho, Ruilong et al ${ }^{9}$ recomiendan cuantificar la captación y establecer valores de corte de SUV para optimizar el rendimiento de la técnica.

$\mathrm{Al}$ analizar los estudios que evalúan la utilidad del PET en NP, se puede reconocer una gran heterogeneidad respecto a la metodología de investigación empleada. Estas diferencias se refieren a la forma de selección de los pacientes, al tipo de NP estudiados (mezclando NP sólidos con NP subsólidos), al tamaño de las lesiones ( $3 \mathrm{~mm}$ hasta $6 \mathrm{~cm}$ ), a las condiciones de la realización del PET/CT, y a los equipos de PET empleados (gamacámaras acondicionadas para PET, PET dedicados, PET/CT, PET/CT + TC contrastada). Más notoria aún es la diferencia que presentan estas publicaciones respecto al criterio para considerar positivo un examen. En un metaanálisis antiguo (publicado el año 2001) que incluyó 40 estudios sobre el valor diagnóstico del PET en NP, en 35\% de estos (14/40) no se explicitó el criterio para definir un PET positivo, y en otro tanto el criterio fue sólo cualitativo ${ }^{10}$. Este tipo de estudios hace muy poco reproducible sus resultados si es que algún investigador que los lee quiere practicarlos en su población, y por lo tanto, carecen de validez externa $^{11}$.

En las publicaciones que emplean cuantificación de captación, hay un relativo consenso en que el algoritmo más utilizado es el Standarized Uptake Value máximo (SUVmax) $)^{9,12}$, que corresponde a la relación: captación/volumen del vóxel de máxima captación. Un valor de corte de SUVmax de 2,5 ha sido mencionado como el más adecuado para separar NP benignos de malignos $^{5,8}$. Sin embargo, un número no despreciable de lesiones malignas presentarán una captación menor a dicho valor, constituyendo falsos negativos de la técnica, que determinarían pacientes erróneamente no biopsiados u operados a tiempo.

El objetivo de este estudio es determinar el rendimiento diagnóstico del PET/CT semicuantitativo en el estudio de NP utilizando el índice de captación SUVmax, y determinar los mejores valores de corte para determinar lesiones benignas y malignas.

\section{Pacientes y Método}

Se revisó la base de datos de $\mathrm{PET} / \mathrm{CT}$ realizados entre los años 2008 y 2015 en nuestra institu- 
ción, seleccionando aquellos estudios solicitados para evaluación de nódulo pulmonar, con revisión de las imágenes y selección de los NP según los siguientes criterios:

Criterios de inclusión: 1) NP sólido $\geq 7 \mathrm{~mm}$ y $\leq 30 \mathrm{~mm}$; 2) Paciente sin antecedente de cáncer reciente (en los últimos 8 años); 3) Paciente sin antecedentes ni sospecha de metástasis pulmonares.

Criterios de exclusión: 1) NP sólido $<7$ $\mathrm{mm}$; 2) NP en vidrio esmerilado puro; 3) NP mixto: con componente en vidrio esmerilado más componente sólido; 4) masa pulmonar (> $3 \mathrm{~cm}$ ); 5) metástasis pulmonares conocidas, 6) antecedentes de neoplasia maligna tratada hace menos de 8 años.

La confirmación de la naturaleza del nódulo se realizó de manera similar a lo descrito en la literatura ${ }^{3,5}$, basándose en cualquiera de estos dos métodos confirmatorios:

1) Histología (tanto en NP malignos como benignos, que por sus características requirieron biopsia).

2) Seguimiento (en NP benignos, que por sus características no requirieron biopsia). Un seguimiento de 2 años o más sin crecimiento fue considerado como signo de benignidad según los criterios aceptados en publicaciones previas ${ }^{1,2}$.

Según estos criterios, los nódulos confirmados se clasificaron como benignos y malignos.

\section{Aspectos técnicos del PET/CT}

El examen PET/CT se realizó en equipo GE Discovery STE 16 canales, $1 \mathrm{~h}$ después de administrar F18-FDG en dosis de $0,1 \mathrm{mCi} / \mathrm{kg}$ por vía endovenosa. Posteriormente a la adquisición del PET y de la tomografía computada de baja dosis para corrección de atenuación, se realizó una TC de tórax contrastada en todos los pacientes (adquisición volumétrica de 1,25 $\mathrm{mm}$ de grosor).

Los pacientes fueron citados en ayunas (al menos $6 \mathrm{~h}$ ). Se realizó hemoglucotest previo en todos los pacientes, inyectando el radiotrazador sólo en aquellos pacientes con glicemias menores a $200 \mathrm{mg} / \mathrm{dl}$. En caso de glicemias mayores se administró suero fisiológico $500-1000 \mathrm{ml}$, hasta alcanzar glicemias adecuadas.

Antes de efectuar el examen, se midió y pesó a todos los pacientes. La dosis inyectada y hora de inyección, dosis residual en la jeringa y hora de medición, peso y talla del paciente, y glicemia fueron ingresados al equipo PET/CT para un adecuado cálculo del SUV según recomendaciones publicadas $^{12}$.

El SUVmax fue medido por dos observadores expertos, dibujando un área de interés automática en cada nódulo. Para esto se utilizaron las imágenes del estudio de PET corregido mediante TC no contrastada de baja dosis ${ }^{12}$. El SUVmax equivale a la actividad máxima de una lesión dividida por el volumen conocido de un vóxel según la fórmula:

\section{SUVmax NP: Actividad máx en la lesión (Bq) / Volumen píxel con actividad máx (ml)}

\section{Actividad inyectada $(\mathrm{Bq})$ corregida por tiempo} decaimiento / Peso paciente (g)

También se registró la captación hepática utilizando índice SUVprom (SUVpromH) en cada paciente. Se calculó el promedio de SUVpromH del grupo total de pacientes (SUVpromHT $=2,4$ ), y se utilizó como factor de normalización común a toda la muestra ${ }^{13}$. Por ejemplo, un SUVmax NP de 2,0 en un paciente con SUV promH de 3,1 resulta en un SUVmax corregido de 1,5 según la fórmula:

$$
\begin{gathered}
\text { SUVmax corregido }= \\
\frac{\text { SUVmax NP x SUVpromHT }}{\text { SUVpromH }}=\frac{2,0 \times 2,4}{3,1}=1,5
\end{gathered}
$$

Todas las cifras de SUVmax expresadas en los resultados corresponden al SUVmax corregido mediante este método.

Se midió y registró el diámetro mayor de los nódulos pulmonares estudiados, utilizando los cortes axiales y reconstrucción multiplanar (MPR) de las imágenes de la TC de tórax contrastada realizada inmediatamente después del $\mathrm{PET} / \mathrm{CT}$.

\section{Estudio estadístico}

Para los efectos de este estudio se consideró el SUVmax corregido para todas las mediciones. Se comparó el SUVmax entre los NP benignos y malignos, y se establecieron los mejores valores de corte de SUVmax utilizando curvas ROC. Se estimó sensibilidad, especificidad, valor predictivo, y likelihood ratio (LR) del SUVmax. La reproducibilidad interobservador fue calculada mediante programa Medcalc Ink utilizando índice Kappa, y comparada mediante curvas de diferencia Bland-Altman. Para estimar diferencias entre SUVmax y diámetro de los nódulos entre los grupos, se utilizó el test de Mann-Whitney. 


\section{Aspectos éticos}

El estudio contó con la aprobación del Comité de Ética de la institución, con acta emitida el 23 de junio de 2016. Los pacientes incluidos en la base de datos firmaron un consentimiento informado respecto a la utilización de sus imágenes de manera anónima para fines de investigación. Para el análisis de datos se protegió la privacidad y confidencialidad de la información de los pacientes del estudio.

\section{Resultados}

\section{Histología de los nódulos pulmonares}

De los 140 nódulos pulmonares incluidos en este estudio, un $40 \%(n=56)$ correspondieron a lesiones benignas (mediana: 65 años, rango 20-83 años, 63,4\% hombres). De éstos, 34 fueron confirmados por biopsia y 22 por seguimiento tomográfico (seguimiento promedio 40,1 meses, rango 24-80 meses). Los diagnósticos histológicos más frecuentes fueron: benigno no especificado $(n=6)$, hamartoma $(n=6)$, granuloma inespecífico $(n=4), y$ COP/BOOP: cryptogenic organizing pneumonial bronquiolitis obliterante con neumonía en organización $(\mathrm{n}=3)$ ( Tabla 1).

Tabla 1.- Histopatología de los nódulos pulmonares benignos

\begin{tabular}{|lc|}
\hline Biopsiados & \\
Benigno no especificado & 6 \\
Hamartoma & 6 \\
Granuloma inespecífico & 4 \\
BOOP & 3 \\
Tuberculosis & 2 \\
Bronquiolitis crónica & 2 \\
Antracofibrosis & 2 \\
Hemangioma esclerosante & 1 \\
Hiperplasia atípica & 1 \\
Teratoma maduro & 1 \\
Fibrosis intersticial & 1 \\
TEP + infarto pulmonar & 1 \\
Sarcoidosis & 1 \\
Paracoccidomicosis & 1 \\
Nódulo reumatoideo & 1 \\
Silicosis & 1 \\
Sin Biopsia Estable (24 meses de observación) & 22 \\
Total & 56 \\
\hline
\end{tabular}

$\mathrm{BOOP}=$ bronquiolitis obliterante con neumonía en organización; TEP = tromboembolismo pulmonar.

Un 60\% $(n=84)$ de los nódulos fueron confirmados como malignos (mediana: 65 años, rango: 30-92 años, 59,5\% hombres). El diagnóstico histológico más frecuente fue adenocarcinoma ( $\mathrm{n}$ $=38)$, metástasis $(\mathrm{n}=12)$, carcinoma escamoso $(\mathrm{n}=8)$, carcinoma de células pequeñas $(\mathrm{n}=8)$ (Tabla 2).

No hubo diferencias significativas en edad ni sexo entre los pacientes con NP benignos y malignos.

Tabla 2. Histología de los nódulos pulmonares malignos

\begin{tabular}{lr} 
Adenocarcinoma & 38 \\
Adenoescamoso & 1 \\
Células pequeñas & 8 \\
Escamoso & 8 \\
Células grandes & 4 \\
Carcinoide & 2 \\
Metástasis & 12 \\
Maligno no Especificado & 11 \\
Total & 84 \\
\hline
\end{tabular}

\section{Tamaño}

Los nódulos benignos presentaron un significativo menor diámetro (mediana $10 \mathrm{~mm}$, rango: 7 - $27 \mathrm{~mm}$ ) que los malignos (mediana: $18 \mathrm{~mm}$, rango 7 - $30 \mathrm{~mm}$ ), con $\mathrm{p}<0,0001$.

El diámetro tuvo valor predictivo de malignidad, con una curva ROC significativa $(\mathrm{p}<0,0001)$ y un área bajo la curva de 0,769 (error estándar: 0,041; 95\% CI: 0,690 a 6,554).

\section{Estudio semicuantitativo (SUVmax)}

Se observó una alta reproducibilidad interobservador en la medición del SUVmax, con índice Kappa: 0,9572 (error estándar: 0,01682; 95\% CI $0,92423$ a 0,99017$)$. Hubo una mayor variabilidad interobservador para valores bajos de SUVmax (Figura 1).

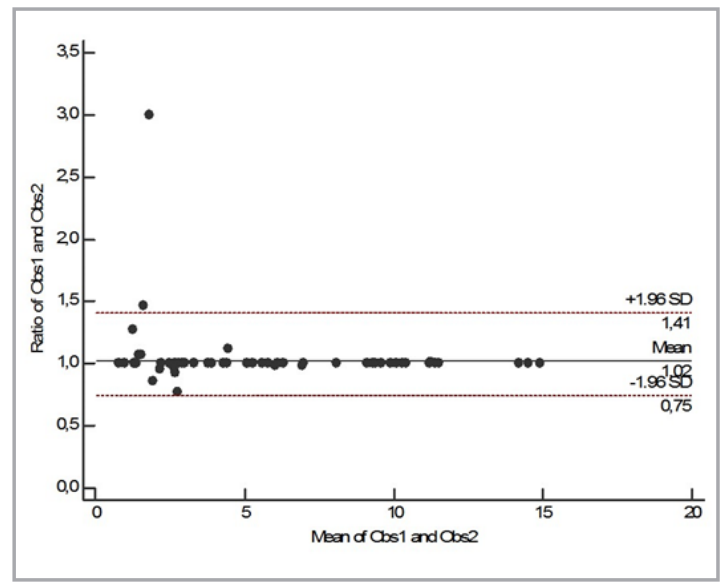

Figura 1. Curva de diferencia Bland-Altman para medición de SUVmax por dos observadores.

Los nódulos malignos presentaron un SUVmax significativamente $(\mathrm{p}<0,0001)$ más alto (mediana: 4,6, rango 0,8 - 17,5) que los nódulos benignos (SUVmax mediana: 1,2, rango: 0,4 - 10,0). 
Los valores de corte de mayor rendimiento diagnóstico encontrados en curvas ROC fueron SUVmax de 1 y 2,5 , con exactitud de $77,1 \%$ para ambos.

La sensibilidad, especificidad, valor predictivo positivo, valor predictivo negativo y Likelihood ratio de los principales valores de corte para el SUVmax se muestran en la Tabla 3.

Tabla 3. Rendimiento diagnóstico para cada valor de corte de SUVmax en nódulo pulmonar sólido

\begin{tabular}{|lccc|}
\hline & SUVmax 1,0 & SUVmax 2,5 & SUVmax 5,0 \\
VP & 83 & 65 & 40 \\
FP & 31 & 13 & 3 \\
VN & 25 & 43 & 53 \\
FN & 1 & 19 & 44 \\
Sensibilidad & $98,8 \%$ & $77,4 \%$ & $47,6 \%$ \\
Especificidad & $44,6 \%$ & $76,8 \%$ & $94,6 \%$ \\
VPP & $72,8 \%$ & $83,3 \%$ & $93,0 \%$ \\
VPN & $96,2 \%$ & $69,4 \%$ & $54,6 \%$ \\
Exactitud & $77,1 \%$ & $77,1 \%$ & $66,4 \%$ \\
LR + & 1,785 & 3,333 & 8,889 \\
LR - & 0,027 & 0,295 & 0,553 \\
\hline
\end{tabular}

VP: Verdaderos positivos; FP: Falsos positivos; VN: Verdaderos negativos; FN: Falsos Negativos; VPP: Valor predictivo positivo; VPN: Valor predictivo negativo; LR: Likelihood ratio.

El valor de corte de SUVmax 1,0 presentó una sensibilidad de $98,8 \%$, valor predictivo negativo de $96,2 \%$, y un LR - de 0,027 .

El valor de corte de SUVmax 2,5 no fue capaz de asegurar razonablemente benignidad con una sensibilidad de $77,4 \%$, VPN de $69,4 \%$ y LR - de 0,295 . Para este valor de corte, el VPP fue de $83,3 \%$ y LR + de 3,333.

El valor de corte de SUVmax 5,0 mostró una especificidad de $94,6 \%$, valor predictivo positivo de $93,0 \%$, y un LR + de 8,889 .

La Figura 2 muestra la distribución de frecuencia de los nódulos malignos y benignos según los principales valores de corte del SUVmax. Al establecer estos 3 valores de corte, se conforman 4 grupos de NP. Como se mencionó, el 96,2\% de los NP con SUVmax $<1,0$ fueron benignos y el 93,0\% de los NP con SUVmax $>5,0$ fueron malignos. De los grupos intermedios, fueron malignos el 50\% de los NP con SUVmax entre 1,1 y 2,5 , y el $71,4 \%$ de los NP con SUVmax entre 2,6 y 5,0 .

El SUVmax presentó alto rendimiento diagnóstico, con una curva ROC significativa $(\mathrm{p}<0,0001)$ y un área bajo la curva de 0,877 (error estándar: 0,0295; 95\% CI: 0,811 a 0,926). El SUVmax fue un predictor de malignidad más

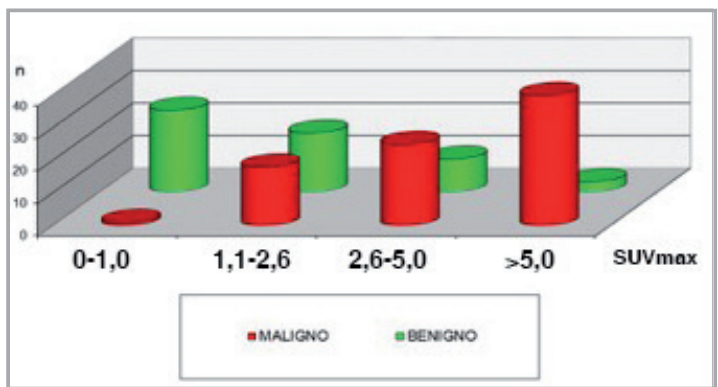

Figura 2. Distribución de frecuencia del SUVmax para distintos valores de corte en nódulos pulmonares malignos y benignos.

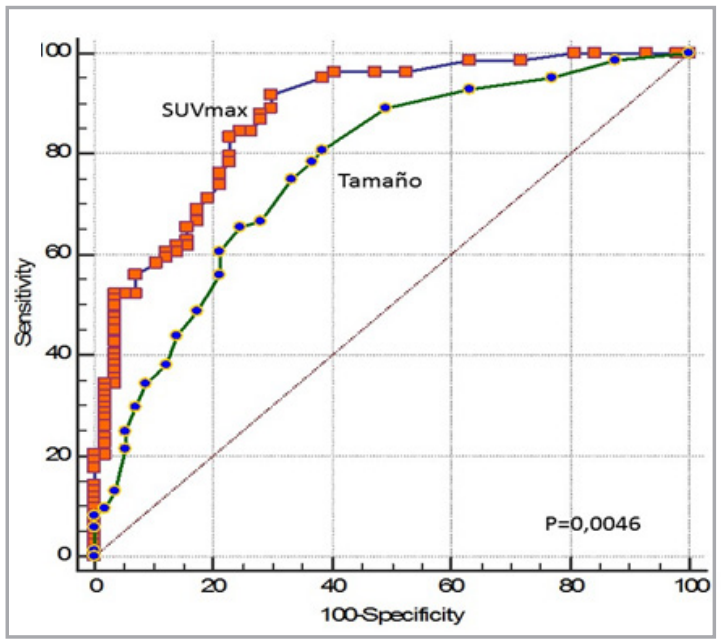

Figura 3. Curva ROC para SUVmax y tamaño (diámetro máximo) de los nódulos pulmonares.

poderoso que el tamaño $(p=0,0046)$. Las curvas ROC comparativas se muestran en la Figura 3.

\section{Discusión}

La utilidad clínica del PET/CT en evaluación de nódulos pulmonares es controversial, y se sabe que para NP de baja captación hay una sobre posición de lesiones benignas y malignas. Sin embargo, es aceptado transversalmente en la literatura que el grado de captación de F18-FDG del NP se asocia a su probabilidad de malignidad, con baja probabilidad para NP no captantes y alta para aquellos de captación intensa ${ }^{5,6,7,8,9}$. Por este motivo sería deseable clasificar en grupos a la población según el grado de actividad de cada lesión, para estratificar riesgos y guiar el manejo. Esto es posible sólo cuantificando la captación del NP.

Por otro lado, algunos autores apoyan la graduación cualitativa de la captación. Un estudio español reciente ${ }^{14}$ introduce un análisis visual comparativo con la actividad del pulmón, me- 
diastino e hígado, graduando la captación en 5 grupos, desde aquellos NP no captantes (captación no mayor al parénquima pulmonar) hasta aquellos de alta actividad (captación marcadamente mayor al hígado), y la proponen por sobre el SUV. Mediante este análisis visual obtienen una sensibilidad de $84 \%$, VPN de $91 \%$ y VPN de $91 \%$, que sería levemente mayor a lo obtenido con SUV (no corregido). No obstante estos resultados, la reproducibilidad de la estimación visual fue moderada (Kappa: 0,79), bastante menor que la descrita para el SUV ${ }^{15}$ y que la obtenida en nuestra muestra (Kappa: 0,95). Esto último restaría validez externa al estudio ${ }^{11}$. Otra limitación del citado trabajo es que incluyen una significativa cantidad de NP subsólidos en su muestra, los que se sabe corresponden a lesiones donde el $\mathrm{PET} / \mathrm{CT}$ no tiene el mismo valor diagnóstico, $\mathrm{y}$ que de hecho representan más de la mitad de los "falsos negativos" de su población.

Aunque aún parece no haber consenso en si cuantificar o no la captación de los NP, la tendencia, al igual que en otros tipos de lesiones o tumores, es a medirla (con el algoritmo que corresponda), lo que permitiría optimizar el rendimiento diagnóstico 9 . Para los NP este algoritmo parece ser el SUV. Así lo demuestran los diferentes metaanálisis recientes disponibles ${ }^{8,9}$, que se basan casi exclusivamente en estudios semi-cuantitativos empleando mayoritariamente el SUVmax. Hay que tener presente que para que el valor obtenido de SUVmax sea confiable y verdaderamente representativo de la actividad real de la lesión, deben considerarse numerosos factores como el peso del paciente, glicemia, tiempo entre la inyección y la adquisición de la imagen $^{12,13}$.

Nuestro estudio intenta establecer la utilidad del PET/CT semicuantitativo en la evaluación de NP midiendo el grado de captación de F18-FDG mediante SUVmax, en una población representativa donde la frecuencia de nódulos malignos es de $60 \%$, muy similar a lo encontrado en la literatura en estudios análogos ${ }^{16}$. Sin embargo, a diferencia de los artículos originales y metaanálisis disponibles, propone valores de corte de SUVmax diferentes al SUVmax de 2,5 mencionado en todas ellas como el más úti1 $1^{3,5,6,7,16}$.

Un SUVmax $\leq 1$ fue encontrado altamente predictivo de benignidad, con un valor predictivo negativo de 96,2\% y Likelihood ratio de 0,027, lo que permitiría evitar procedimientos invasivos con un alto grado de seguridad. Estos pacientes no debieran biopsiarse, y se propone control con
TC de baja dosis como una buena alternativa para evaluar eventuales cambios de tamaño de la lesión en el tiempo.

Por otro lado, un SUVmax $\geq 2,5$ fue altamente predictivo de malignidad, y el estudio cito/ histológico posiblemente sea la conducta más razonable, sabiendo que un porcentaje muy bajo de estos casos resultarán finalmente benignos ( $17 \%$ en nódulos con SUVmax $\geq 2,5$ y $7 \%$ en nódulos con SUVmax $\geq 5$ ). Es más, un NP con captación $\geq 5,0$ debiera ser considerado como de altísima probabilidad de malignidad, orientando a una resección quirúrgica de manera directa, probablemente con criterio oncológico.

El grupo de nódulos con captación intermedia entre 1,0 y 2,5 es posiblemente el que plantea los desafíos más importantes respecto a la conducta a seguir. La mitad de ellos resultará maligno si se biopsia, es decir, tiene una probabilidad intermedia de ser cáncer. Es posible que en estos casos sea de utilidad considerar otros factores antes de tomar una decisión de qué conducta seguir, como por ejemplo: edad, antecedentes de tabaco, ubicación en lóbulos superiores y espiculación del nódulo, que se sabe se asocian a malignidad ${ }^{2,17}$. El análisis de dichos factores no formó parte de los objetivos de este trabajo, sin embargo, queda planteado como tema para futuras investigaciones.

Nuestro estudio emplea el concepto de SUVmax corregido, es decir corregir la captación del nódulo de acuerdo a una estructura con captación estándar que pueda compararse entre los pacientes. Según las recomendaciones internacionales ${ }^{13}$, la estructura utilizada fue el hígado, considerando que es uno de los órganos con intensidad de captación menos variable en una población de individuos distintos. Dicha normalización proporciona un valor de SUVmax más confiable y más homogéneo entre una población per se heterogénea. Esta corrección del SUVmax se encarga de disminuir la variabilidad que significa el distinto peso, talla, composición corporal de grasa, niveles de glicemia ya mencionados, y otros factores biológicos intrínsecos como la distribución fisiológica del F18-FDG propia de cada individuo ${ }^{13}$, y es vital en casos de dosis residual en el sitio de inyección, hecho no poco frecuente en estudios de medicina nuclear, caso en que se tiende a subestimar el índice SUV por que no toda la dosis administrada alcanza el torrente sanguíneo para su distribución en el organismo.

Como se mencionó antes, este estudio se limitó a evaluar la utilidad del SUVmax como factor 
individual en el estudio de nódulos pulmonares sólidos, y establecer sus mejores valores de corte para diferenciar lesiones benignas de malignas. Por lo tanto, no se estudió ninguna de las otras características de los NP, ni se estableció ninguna relación con factores de riesgo como el tabaco, edad, antecedentes personales o familiares, elementos que debieran ser siempre considerados al momento de evaluar a un paciente con $\mathrm{NP}^{1}$.

Otra de las limitaciones de este estudio es la subestimación del real número de pacientes con lesiones benignas, que al mostrar baja captación al PET/CT no llegaron a biopsiarse, y que se controlaron fuera de nuestra institución, sin poder enrolarse en este estudio por no tener la información del seguimiento. Esta situación es inversa en la población con lesiones de alta captación de F18-FDG, que en alta proporción se realizaron resección y/o seguimiento postquirúrgico, pudiendo acceder a toda la información necesaria para formar parte del estudio. A pesar de esto, la población estudiada parece representativa en edad, sexo y en cuanto a proporción de nódulos malignos, con datos similares a otras series ${ }^{7,16}$.

La pérdida del seguimiento de pacientes con nódulos benignos constituye un sesgo de nuestro estudio, que incide en una probable subestimación del poder del PET/CT respecto a evitar toracotomías innecesarias, ya que es posible que el número de toracotomías o biopsias por punción evitadas gracias al PET/CT sea bastante mayor a los casos documentados en nuestra serie. Por otro lado, la prevalencia de lesiones malignas pudiera estar sobreestimada por esta misma razón, lo que debe ser considerado al evaluar el valor predictivo del PET/CT, principalmente el VPN ya que el detrimento se produciría especialmente en los verdaderos negativos que no se llegaron a confirmar.

\section{Conclusión}

E1 PET/CT presenta un alto rendimiento diagnóstico en estimar la naturaleza de un nódulo pulmonar sólido utilizando el índice SUVmax corregido por la captación hepática. Un valor de SUVmax $\leq 1$ es altamente predictivo de nódulo benigno, y un valor de $\mathrm{SUVmax} \geq 2,5$ de nódulo maligno. En nódulos con valores de SUVmax entre 1,0 y 2,5 el PET/CT no es capaz de caracterizar eficientemente los NP. En estos casos sería necesario contar con información adicional como antecedentes clínicos y características tomográficas del nódulo para ayudar a determinar el manejo adecuado.

\section{Bibliografía}

1.- BUENO J, LANDERAS L, CHUNG JH. Updated Fleischner Society Guidelines for Managing Incidental Pulmonary Nodules: Common Questions and Challenging Scenarios. Radiographics 2018; 38:1337-50.

2.- GOULD MK, DONINGTON J, LYNCH WR, MAZZONE PJ, MIDTHUN DE, NAIDICH DP, WIENER RS. Evaluation of individuals with pulmonary nodules: when is it lung cancer? Diagnosis and management of lung cancer, 3rd ed: American College of Chest Physicians evidence-based clinical practice guidelines. Chest 2013; 143(5 Suppl): e93S-e120S.

3.- ALPERT JB, LOWRY CM, KO JP. Imaging the Solitary Pulmonary Nodule. Clin Chest Med 2015; 36: 161-78.

4.- JADVAR H, ALAVI A, GAMBHIR SS. 18F-FDG Uptake in Lung, Breast, and Colon Cancers: Molecular Biology Correlates and Disease Characterization. J Nucl Med 2009; 50: 1820-7.

5.- AL-AMERIA A, MALHOTRAB P, THYGESENC H, PLANTD PK, VAIDYANATHANE S, KARTHIK S, et al. Risk of malignancy in pulmonary nodules: A validation study of four prediction models. Lung Cancer 2015; 89: 27-30.

6.- $\quad$ SHINGO IWANOA S, ITO S, TSUCHIYA K, KATO $\mathrm{K}$, NAGANAWA S. What causes false-negative PET findings for solid-type lung cancer? Lung Cancer 2013; 79: 132-6.

7.- $\quad$ LI S, ZHAO B, WANG X, YUB J, YANA S, LVA C, et al. Overestimated value of 18F-FDG PET/CT to diagnose pulmonary nodules: Analysis of 298 patients. Clinical Radiology 2014;69:e352-e357.

8.- LI ZZ, HUANG YL, SONG HJ, WANG YJ, HUANG Y. The value of $18 \mathrm{~F}-$ FDG-PET/CT in the diagnosis of solitary pulmonary nodules: A meta-analysis. Medicine (Baltimore) 2018; 97: e0130.

9.- RUILONG Z, DAOHAI X, LI G, XIAOHONG W, CHUNJIE W, LEI T. Diagnostic value of $18 \mathrm{~F}-\mathrm{FDG}$ $\mathrm{PET} / \mathrm{CT}$ for the evaluation of solitary pulmonary nodules: a systematic review and meta-analysis. Nucl Med Commun 2017;38:67-75.

10.- GOULD MK, MACLEAN CC, KUSCHNER WG, RYDZAK CE, OWENS DK. Accuracy of positron emission tomography for diagnosis of pulmonary nodules and mass lesions: a meta-analysis. JAMA 2001; 285: 914-24.

11.- MANTEROLA C, OTZEN T. Análisis Crítico de la Literatura Biomédica. Int J Morphol 2014; 32:599-607.

12.- BOELLAARD R. Standards for PET Image Acquisition and Quantitative Data Analysis. J Nucl Med 2009; 50: 11S-20S. 
13.- ADAMS MC, TURKINGTON TG, WILSON JM, WONG TZ. A Systematic Review of the Factors Affecting Accuracy of SUV Measurements. AJR 2010; 195 : 310-20.

14.- GARCÍA-VELLOSO MJ, BASTARRIKA G, DETORRES JP, LOZANO MD, SÁNCHEZ-SALCEDO $\mathrm{P}$, SANCHO L, et al. Assessment of indeterminate pulmonary nodules detected in lung cancer screening: Diagnostic accuracy of FDG PET/CT. Lung Cancer 2016; 97: 81-6.

15.- NAHMIAS C, WAHL LM. Reproducibility of Standardized Uptake Value Measurements Determined by
18F-FDG PET in Malignant Tumors. J Nucl Med 2008; 49: 1804-8.

16.- DEPPEN SA, BLUME JD, KENSINGER CD, MORGAN AM, ALDRICH MC, MASSION PP, et al. Accuracy of FDG-PET to Diagnose Lung Cancer in Areas With Infectious Lung Disease:A Meta-analysis. JAMA 2014; 312: 1227-36.

17.- GOULD MK, SANDERS GD, BARNETT PG, RYDZAK CE, MACLEAN CC, MCCLELLAN MB, et al. Cost-effectiveness of alternative management strategies for patients with solitary pulmonary nodules. Ann Intern Med 2003; 138: 724-35.
Correspondencia a:

Dr. David Ladrón de Guevara Hernández

Clínica Las Condes, Servicio de Radiología $1^{\circ}$ piso.

Lo Fontecilla 441, Las Condes.

e-mail: dlg@clc.cl 